\title{
Retard de croissance intra-utérin et déficit en néphrons
}

\author{
Claudie Merlet-Bénichou, Bruno Leroy, Thierry Gilbert, \\ Martine Lelièvre-Pégorier
}

\section{Summary \\ Intrauterine growth retardation and inborn nephron deficit}

The number of nephrons at birth is normally quite fixed in any species. Severe renal hypoplasia is readily detected because of functional insufficiency, but moderate inborn nephron deficits may exist, yeat remain undetected. A systematic anatomical study revealed a large quantitative variation of the number of nephrons at birth in man (600000-1400000). Inborn nephron deficits were found to correlate very strongly with birth weight in the rat. The same correlation exists in man : intrauterine growth retardation may be a major cause of inborn nephron deficit, pointing to the possible involvement of common growth factors responsible for fetal development and determination of the number of kidney functional units. Inborn nephron deficit may also proceed from drug toxicity, as described for gentamicin. The deficit is usually compensated for by hypertrophy of the remnant nephrons and hyperfiltration. This may lead to accelerated glomerulosclerosis, as shown in the rat, and be responsible for subsequent proteinuria, renal insufficiency and arterial hypertension. Epidemiologic and experimental studies are now necessary to verify this hypothesis and analyze the underlying mechanisms leading from intrauterine growth retardation to hypertension in the adulthood.

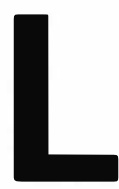
a néphrogenèse, lorsqu'elle se déroule normalement, aboutit à un nombre relativement fixe de néphrons pour une espèce donnée. Chez l'homme, ce nombre est en moyenne de 1 million par rein. Des déficits congénitaux en néphrons sont décrits, mais ils ne sont décelés que lorsqu'ils s'intègrent dans un syndrome malformatif ou bien lorsque, isolés mais sévères, ils sont à l'origine de troubles fonctionnels importants [1, 2]. Dans le cas de l'hypoplasie oligoméganéphronique bilatérale, par exemple, le nombre de néphrons peut être réduit à 200000 par rein [3]. Les données de la littérature montrent que, indépendamment de ces déficits sévères, le nombre de néphrons chez l'homme varie considérablement, de 600000 à 1400000 environ $[4,5]$. Il existe donc des déficits de moindre sévérité, mais leur fréquence est difficile à déterminer car la plupart d'entre eux demeurent ignorés. De tels déficits, bien que non explorés, sont généralement considérés comme n'ayant pas de conséquence fonctionnelle. La question a été cependant posée de leur rôle comme facteur favorisant l'hypertension, et de leur influence sur la progression des lésions en cas de maladie rénale surajoutée [6].

Une relation entre le poids de naissance et le nombre de néphrons a été récemment mise en évidence. Elle montre qu'un retard de croissance intra-utérin s'accompagne d'un déficit en néphrons. Celui-ci est définitif, puisque, contrairement à d'autres organes, le rein n'a pas, après la naissance, la capacité d'engendrer de nouvelles unités fonctionnelles, leur nombre étant définitivement acquis au cours de la vie fotale. La mise en évidence d'une telle corrélation entre poids de naissance et nombre de néphrons fait apparaître l'ampleur de la population éventuellement concernée par un déficit néphronique congénital, puisqu'en France, par exemple, près de 80000 enfants naissent chaque année avec un retard de croissance intrautérin plus ou moins sévère ; elle devrait conduire à faire figurer désormais l'hypotrophie à la naissance parmi les facteurs de risque de progression des maladies rénales; enfin, elle ouvre des perspectives nouvelles d'interprétation dans des enquêtes épidémiologiques récentes qui ont mis en évidence une relation entre le poids de naissance et la pression artérielle dans l'enfance et à l'âge adulte [7, 8]. Des facteurs nutritionnels et hormonaux ont été proposés pour expliquer cette relation $[9,10]$, mais on peut se demander si le déficit en néphrons qui semble accompagner tout retard de croissance intra-utérin n'explique pas en partie le développement ultérieur de l'hypertension chez les enfants de petit poids [11].

\section{Relation entre le retard de croissance intra-utérin et le nombre de néphrons}

La cause et les mécanismes des oligonéphronies isolées, quelle qu'en soit la séverité, sont totalement inconnus. Il a été montré récemment que l'exposition in utero à la gentamicine induit un déficit en néphrons d'environ $20 \%$ en moyenne chez le rat $[12,13]$. L'examen en microscopie électronique de reins de rats exposés in utero à la gen- 
tamicine ainsi que des études in vitro sur explants de reins embryonnaires mis en culture montrent que le déficit peut résulter d'un effet direct de l'antibiotique sur le rein fœtal $[14,15]$. Cependant, l'exposition in utero à la gentamicine induit également un retard de croissance plus ou moins sćvère des fœtus. Plus le retard de croissance est important, plus le déficit en néphrons est lui-même important, ce qui suggérait que le déficit en néphrons observé était en partie dû au retard de croissance [13].

Les données de la littérature sur l'effet d'un retard de croissance sur le développement du rcin sont rares et contradictoires [16, 17]. Nous avons donc étudié deux modèles classiques de retard de croissance intra-utérin obtenus chez le rat, l'un par ligature partielle d'une artère utérine maternelle au 17 e jour de la gestation, l'autre par carence protéique à partir du 8 e jour de la gestation. Le dénombrement des néphrons sur rein enticr après macération acide de l'organe montrc qu'un retard de croissance est dans tous les cas associé à un déficit en néphrons [18]. Dans le cas d'unc hypotrophie sćvèrc, le déficit peut atteindrc plus de $50 \%$ du nombre normal de néphrons. Lc fait remarquable cst qu'il existe unc corrélation linéaire significative entre le nombre de néphrons et le poids à la naissance (figure 1). Cette étroite corrélation, qui existe même dans un intervallc de poids normal, pourrait suggérer que les mêmes facteurs qui contrôlent la croissancc fœtale, contrôlent les mécanismes qui déterminent le nombre de néphrons. Une voie de recherche prometteuse parmi d'autres pour mieux comprendre le mécanisme des déficits en néphrons associés au retard de croissance intra-utérin concernc le rôle possible des facteurs de croissance, notamment des insulin like growth factors (IGF) fœtaux et de leurs protéines de liaison spécifiques, qui semblent être, avec l'insuline, les principaux régulateurs endocriniens de la croissance prénatale [19]. L'implication de ces mêmes facteurs dans le contrôle de la néphrogenèse a été récemment démontrée in vitro [20].

L'analyse rétrospective de coupcs dc reins d'enfants nés à terme et décédés en réanimation à l'hôpital ArmandTrousseau (Paris, Francc) sur une période de dix ans, a suggéré que,

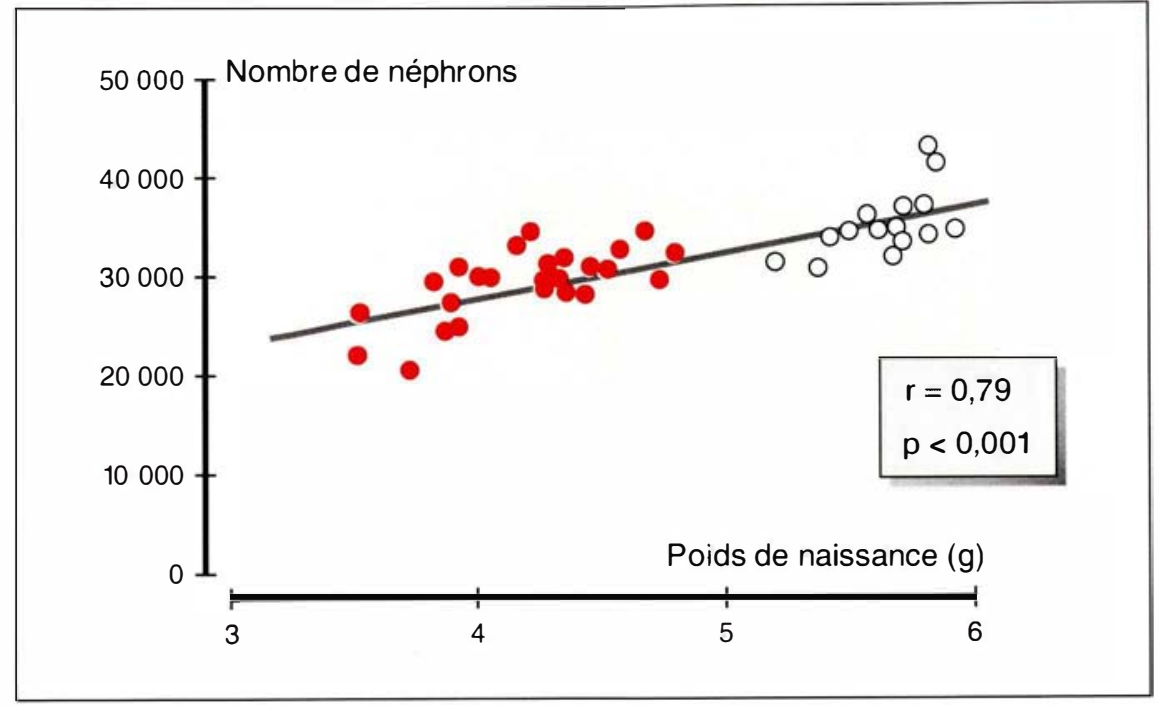

Figure 1. Nombre de néphrons en fonction du poids de naissance chez des rats nés de mères ayant reçu un régime normal (témoins : $\bigcirc$ ou carencé en protéines (hypotrophes: O) pendant la gestation (résultats non publiés, C. Merlet-Bénichou, T. Gilbert, M. Muffat-Joly, M. Lelièvre-Pégorier et B. Leroy).

chez l'homme également, un retard de croissance intra-utérin s'accompagne d'un déficit en néphrons [21]. D'autres autcurs ont rapporté un résultat identique, dans unc étude prospective utilisant aussi du matériel histologique [22]. Enfin, unc étude prospective multicentrique nous permettant de dénombrer lcs néphrons sur rein entier après macération acide de l'organc prélevé à l'autopsie, nous a pcrmis d'établir qu'il cxistait chez l'homme aussi une corrćlation significative entre le nombre de nćphrons et le poids de naissance (figure 2).

Toutcfois, si un retard de croissancc intra-utérin scmble s'accompagner d'unc oligonéphronie, il est clair que toute oligonéphronie n'est pas associéc à un retard de croissance intra-utérin Dans l'hypoplasie rénale oligoméganéphroniquc, une hypotrophie à la naissance est parfois constatée, mais elle n'est cependant pas la règle [23, 24]. L'hypotrophie n'est sans doute pas lc seul facteur responsable d'oligonéphronic. Les donnécs obtenues chez l'animal montrent que l'exposition in utero à certains médicaments peut également perturber la néphrogenèsc sans qu'il y ait hypotrophic associée. Le rôle d'autres facteurs insoupçonnés à ce jour ne pcut évidemment être exclu. Enfin, il est permis de penser que les effets de plusieurs facteurs puissent s'additionner. Ainsi, dans le modc̀le de déficit en néphrons acquis in utero par exposition à la gentamicine, nous avons montré à l'aide de traitements de courte durée n'entraînant pas d'hypotrophie, quc l'cffet du retard de croissance sur la néphrogenèse est le plus souvent modéré, mais qu'il s'ajoute à l'cffet direct de l'antibiotique sur le rcin fœtal, de tellc sorte qu'un déficit global de plus de $50 \%$ peut être observé [25].

\section{Conséquences à long terme des déficits en néphrons acquis in utero}

Une réduction du nombre de néphrons, qu'elle soit acquisc avant ou après la naissance, entraînc une adaptation compensatrice caractérisće par l'hypertrophie ct l'hyperfiltration des nćphrons restants. Dans un premicr temps, cette adaptation apparaît bénéfique, puisqu'cllc tend à restaurer une fonction rénale globale adéquate, mais à terme elle s'avère néfaste puisqu'elle entraîne une glomérulosclérose [26]. Pour certains auteurs, les lésions glomérulaires sont ducs principalement à l'hypcrfiltration et à l'hypertension capillaire qui cn résultent [27]. Pour d'autres auteurs qui ont pu établir une corrélation entre le degré de sclérose et le volume glomérulaire, mais pas avec 
les modifications hémodynamiques, la physiopathologie des lésions mettrait en jeu différents facteurs agissant commc promotcurs de la croissance glomérulaire [28]. On sait ćgalement que, chez l'animal, l'hypertrophie compensatrice des néphrons restants cst d'autant plus importante qu'il est jeune lorsque survient la réduction néphronique [29]. Chez l'homme, les déficits en néphrons congénitaux très sćvères, comme l'oligoméganéphronie, conduisent à l'insuffisance rénalc terminale dans l'enfance ou l'adolescence [23]. Dans les déficits moycnnement sévères, comme l'agénésie unilatérale, le risque d'apparition d'une hypertension, d'une protéinurie et d'une insuffisance rénale cst augmenté, d'après la plupart des auteurs [30-33]. Dans ces études, unc biopsie effectuée chez 13 patients a montré dans tous les cas des lésions glomérulaires importantes [31, 32, 34, 35]. Ce domainc d'investigation manque cependant encore d'études prospectives. Celles-ci devraient maintenant être possibles grâce au dévcloppcment de l'échographic obstétricale, permettant un dépistagc plus systématique d'anomalies du développement rénal. Quant aux déficits de moindre sévérité, ils demeurent inexplorés en clinique car ignorés. Faute de modèles animaux adéquats, on manque également de données expérimentales sur les conséquences de ces déficits.

Les modèles expérimentaux de réduction nćphronique originaux mentionnés plus haut, qui ont l'avantage d'être non invasifs, et qui surtout préscntent un degré de déficit variable, prévisible à partir du poids de naissance, nous permettent actuellement d'étudier les consćquences de déficits acquis in utero, en particulier lcs déficits modérés. Lc suivi sur une période d'un an de rats nés de mères traitées par la gcntamicine, a déjà fait apparaître qu'un déficit en néphrons de $20 \%$ seulement est suffisant pour accélérer le développement des lésions glomérulaires sclérotiques liécs à l'âge dans l'cspècc ćtudiée [36]. Sur cette période, la diminution du débit de filtration glomérulaire, bien que significative, est modérée. L'absencc de lésions précoces chez quelques animaux qui, malgré l'exposition in utero à la gentamicine, n'avaicnt pas de déficit en néphrons, suggc̀re que lorsqu'un déficit est présent, lui seul est responsable de l'appa$\mathrm{m} / \mathrm{s} n^{\circ}$ 6-7 vol. 9, juin-juillet 93 rition précoce des lésions, et non l'accumulation prolongéc d'antibiotique dans lc tissu rénal. En effet, chez des rats nés avec une oligonéphronie modérée de mères carencécs en protéines, la fréquence des lésions glomérulaircs sclérotiques est significativement augmentée dès l'âge de trois mois [37]. Quant à la physiopathologie dc l'hypertension consécutivc à un déficit congénital en néphrons, elle ferait intervenir unc rétention sodée, résultant de la diminution de la surface de filtration glomérulaire, avec pour conséquence une augmentation du volume plasmatique. L'élévation de la pression artérielle, qui augmente la natriurc̀se, représenterait alors un mécanisme compensateur destiné à rétablir la volćmic [6]. Cependant, l'hypothèse selon laquelle un déficit congénital en néphrons modéré pourrait également favoriser l'hypertension repose principalement sur des donnćes cliniques et cxpérimentales qui concernent des déficits d'au moins $50 \%$ de la massc rénalc [6]. Dc plus, les modèles expérimentaux généralement cités sont criticables en ce sens que la méthode utilisée pour réduire le nombre de néphrons (ligature de branches de l'artère rénale), et non la réduction néphronique, pourrait engendrer par elle-même l'hypertension [38]. Une réduction

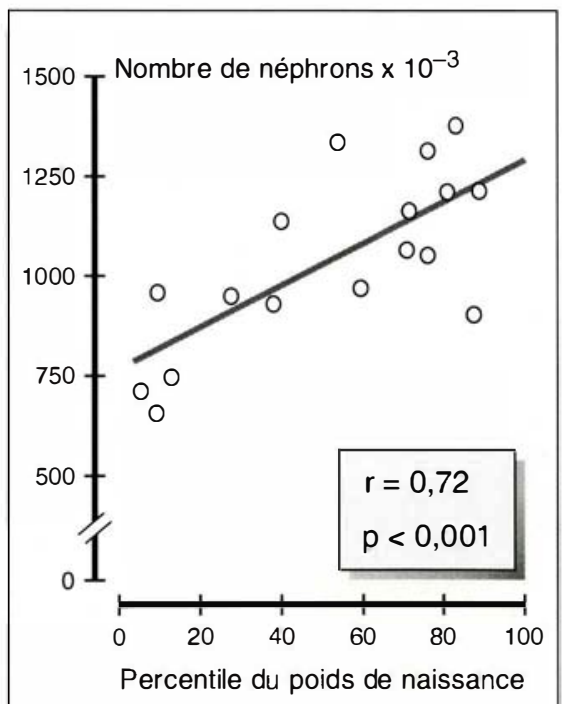

Figure 2. Nombre de néphrons chez l'homme en fonction du poids de naissance /résultats non publiés, B. Leroy, T. Gilbert, M. Lelièvre-Pégorier, G. Chéron, C. Rambaud, C. Nessmann, A.-L. Delezoide et C. Merlet-Bénichou). néphronique équivalente induite par l'cxcision de tissu rénal peut entraîner une hypertension, mais celle-ci, lorsqu'ellc est présentc, reste modéréc et d'apparition tardive [39]. D'autres données, plus pertinentes mais peu nombreuses, sont également citćes à l'appui de cettc hypothèse : il a été montré que différentes souches de rats dans lesquelles la susceptibilité à l'hypertension peut ĉtre augmentée par un régime hypersodé, ont un déficit en néphrons d'environ $15 \%$ [6] ; par ailleurs, une étude ancienne analysant les reins prélevés à l'autopsie chez 65 patients, a mis en évidence chez 28 d'entre eux un nombrc de néphrons inférieur à 700000 [40]. Chez ces 28 paticnts, la pression artérielle systolique mesuréc avant la mort était dans tous les cas supérieure à $150 \mathrm{mmHg}$. Chez les 37 patients ayant plus de 700000 néphrons, neuf seulement avaient une hypertension. Dans les modèles de déficit en néphrons acquis in utero que nous avons étudiés, la pression artérielle moyenne, mesurée sous anesthésie chez quelques animaux, n'était pas modifiée. Unc étude chez l'animal éveillé est en cours.

\section{Conclusion}

Il est maintenant clairement ćtabli que chez le rat le nombre de néphrons est corrélé au poids de naissance. La même corrélation a été retrouvée dans les premiers résultats obtenus chez l'homme; certains sujets ayant un retard de croissance intra-utérin naissent avec un déficit en néphrons modéré. Il est aussi démontré chez le rat qu'un déficit nćphronique congénital de $20 \%$ suffit à accélérer l'apparition de la glomérulosclérose liée à l'âge. Ces données ouvrent de nouvcaux axes de recherche et devraient être prises en compte dans le suivi des néphropathies et des maladies susccptibles d'entraîner une glomérulosclérose, et donc de diminuer encore le capital de néphrons, comme par exemple le diabète, l'hypertension artérielle ou la drépanocytose homozygote. Elles devraicnt être prises en compte également dans l'étude des effets rénaux de ccrtains médicaments, en particulicr l'hormone de croissance biosynthétique utiliscee actuellement dans le cadre d'essais thérapeutiques chez des enfants présentant un retard de croissance à la 
naissance et qui n'ont pas eu de rattrapage statural. Enfin, ces données obtenues pour le rein posent le problème plus général des conséquences d'un retard de croissance intra-utérin qui non seulement diminue le poids de nombreux organes, mais qui peut aussi, pour certains d'entre eux, diminuer de façon définitive le nombre d'unités fonctionnelles, ayant ainsi des conséquences néfastes, pas nécessairement immédiates, sur la fonction de ces organes.

Plus spéculative est, à l'heure actuelle, l'hypothèse d'une relation entre le poids de naissance, la réduction néphronique modérée et l'hypertension à l'âge adulte. Compte tenu de l'importance des populations concernées, il apparaît nécessaire d'entreprendre des travaux expérimentaux et épidémiologiques permettant de vérifier cette hypothèse et d'analyser les mécanismes qui sous-tendent cette relation

\section{RÉFÉRENCES}

1. Habib R, Courtccuisse V, Mathicu H Royer P. Un type anatomo-clinique particulicr d'insuffisance rénale chronique de l'cnfant : l'hypoplasic oligonćphronique congénitalc bilatéralc. J Urol Nephrol 1962 ; 68 : 139-43.

2. Bernstein J, Mcycr R. Sorne speculations on the nature and significance of developmentally small kidncys (renal hypoplasia). Nephron $1964 ; 1$ : 137-43.

3. Fetterman $\mathrm{CH}$, Habib R. Congenital bilateral oligonephronic renal hypoplasia with hypertrophy of nephrons (oligoméganéphronic). Studics by microdissection. Am J Clin Pathol $1969 ; 52$ : 199-207.

4. Dunnil MS, Halley W. Some obscrvations on the quantitative anatomy of the kidncy. J Pathol 1973 ; 110 : 113-21.

5. McLachlan MSF, Guthric JC, Anderson CK, Fulker MJ. Vascular and glomerular changes in the aging kidncy. J Pathol $1977 ; 121: 65-78$

6. Brenner BM, Garcia IL, Anderson S Glomeruli and blood pressure. Less of onc, more of the other? Am J Hypertension 1988 ; 1 : 335-47

7. Barker IJP, Osmond C, Golding J, Kuh I, Wadsworth MEJ. Growth in utero, blood pressurc in childhood and adult life, and mortality from cardiovascular discasc. $\mathrm{Br} \mathrm{Med}$ J $1989 ; 298: 564-7$.

8. Whincup PH, Cook IG, Shaper AG. Early influences on blood pressurc : a study of children aged 5-7 years. Br Med J 1989 ; 299 : 587-91.

9. Edwards CRW, Bencdiktsson R, Linsay RS, Scckl JR. Disfunction of placental glucocorticoid barricr : link between fetal environment and adult hypertension. Lancet 1993 ; 341: 355-7.
10. Barker DJP, Gluckman PI), Godfrey KM, Harding JE, Owens JA, Robinson JS. Fetal nutrition and cardiovascular discasc in adult lifc. Lancet $1993 ; 341$ : 938-41 11. Merlet-Bénichou C, Leroy B, Gilbert T, Lclièvre-Pégorier M. Placental insufficiency and its effect on the fetus and adult discase. Lancet 1993 ; 341 : 827-8

12. Mallié JP, Gérard H, Gérard A. Gentamicin administration to pregnant rats : effect on renal development in utero. Dev Pharmacol Ther $1984 ; 7$ (suppl 1) : 89-92.

13. Gilbert T, Lclic̀vre-Pégorier M, Maliénou R, Mculcmans A, Mcrlet-Bénichou C. Effects of prenatal and postnatal exposure to gentamicin on renal differentiation in the rat. Toxicol 1987 ; 43 : 301-13.

14. Gilbert T, Nabarra B, Merlet-Bénichou C. Light and electron microscopic analysis of the kidncy in newborn rats exposed to gentamicin in utero. Am J Pathol $1988 ; 130$ : 33-43.

15. Cavalier S, Morcau E, Gilbert T, MerletBénichou C. In vitro effect of gentamicin on renal differentiation in the rat. Renal Physiol Biochem 1992 ; 15 : 196.

16. Zeman FJ. Effects of maternal protein restriction on the kidncy of the newborn young of rats. I Nutr 1968; 94 : 111-6.

17. Sandstrom DJ, Troy JL, Brenner BM, Rennkc HG. Effect of maternal dictary protein content on nephron development in the rat and progressive glomerulosclcrosis following nephrectomy. I Am Soc Nephrol 1990 ; 1 : 295. 18. Merlet-Bćnichou $\mathrm{C}$, Lelièvre-Pégorier $\mathrm{M}$, Gilbert T, Muffat-Joly M, Lcroy B. Intrauterine growth retardation (IUGR) and inborn nephron deficit in the rat. $J A m$ Soc Nephrol 1992 ; 3 : 524.

19. Brauner R, de Zegher F. Croissance et maturation fatales. médecine/sciences 1993 ; 9 : 271-6.

20. Hammerman MR, Rogers SA, Ryan G. Growth factors and metancphrogenesis. Am J Physiol 1992 ; 262 : 523-32

21. Lcroy B, Josset P, Morgan G, Costil J, Merlet-Bénichou C. Intrautcrinc growth retardation (IUGR) and nephron deficit : preliminary study in man. Pediatr Nephrol $1992 ; 6$ : 3 .

22. Hinchliffe SA, Lynch MR, Sargent PH, Howard CV, Van Velzen I). The effect of intrautcrinc growth retardation on the development of renal nephrons. Br J Obstet Gynaecol 1992 ; 99 : 296-301.

23. Royer P, Habib R, Lcclerc F. L'hypoplasic rénale bilatéralc avec oligoméganćphronic. $3^{\text {rd }}$ International Congress of Nephrology. Bascl/New York: Karger, 1967 : 251-75.

24. Schcinman JI, Abclson HT. Bilateral renal hypoplasia with oligoncphronia. J Pediatr 1970 ; 76 : 369-76.

25. Gilbert T, I,clièvre-Pégorier M, MerletBénichou C. Immediatc and long-term renal effects of fetal exposure to gentamicin. Pediatr Nephrol 1990 ; 4: 445-50.

26. Brenner BM. Nephron adaptation to renal injury or ablation. Am J Physiol 1985 ; 249 : 324-37.

27. Brenner BM. Hemodynamically mediated glomerular injury and the progressive nature of kidncy discasc. Kidney Int 1983 ; 23 647-55.

28. Fogo A, Ichikawa I. Evidence of central rolc of glomerular growth promoters in the devclopment of sclerosis. Semin Nephrol 1990 9 : 329-42.

29. Kaufman JM, Hardy R, Hayslett JP Age-dependent characteristics of compensatory renal growth. Kidney Int 1975; 8 : 21-6.

30. Argucso LR, Ritchey ML, Boylc ET, Milliner ISS, Bergstralh EJ, Kramer SA. Prognosis of patients with unilateral renal agenesis. Pediatr Nephrol 1992 ; 6 : 412-6.

31. Rugiu C, Oldrizzi L, Lupo A, Valvo E, Loschiavo C, Tessitorc N, Gammaro L, Ortalda V, Fabris A, Panzetta G, Maschio G. Clinical features of patients with solitary kidncys. Nephron 1986; 43 : 10-5.

32. Kiprov DI), Colvin RB, McCluskcy RT Focal and segmental glomerulosclerosis and proteinuria associated with unilateral renal agcncsis. Lab Invest 1982 ; 46 : 275-81.

33. Wikstad I, Celsi G, Larsson L, Herin P, Aperia A. Kidncy function in adults born with unilateral renal agenesis or nephrectomized in childhood. Pediatr Nephrol 1988; 2 : 177-82. 34. Thorner PS, Arbus GS, Celermajer IDS, Baumal R. Focal segmental glomerulosclerosis and progressive renal failure associated with a unilateral kidncy. Pediatrics 1984; 73 : 806-10.

35. Bhathena IDB, Julian BA, McMorrow RG, Bachler RW. Focal sclerosis of hypertrophicd glomeruli in solitary functioning kidncys of humans. Am J Kidney Dis 1985 5 : 226-32.

36. Gilbert T, Lclic̀vre-Pćgoricr M, MerletBénichou C. Long-term effects of mild oligonephronia induced in utero by gentamicin in the rat. Pediatr Res 1991; 30 : 450-6.

37. Gilbert T, I clic̀vre-Pégoricr M, MerletBénichou C. Glomerular damage in adult rat born with mild oligonephronia of protcindeprived mothers. J Am Soc Nephrol $1990 ; 1$ : 629 (abstr).

38. Meyer TW, Rennke HG. Progressive glomerular injury after limited renal infarction in the rat. Am J Physiol 1988 ; 254 : 856-62. 39. Terzi F, Bcaufils H, Laouari I), Burtin M, Klcinknecht C. Renal effect of antihypertensive drugs depends on sodium dict in the excision remnant kidncy model. Kidney Int $1992 ; 42$ : 354-63.

40. Hayman JM, Martin J, Miller M. Renal function and the number of glomeruli in the human kidncy. Arch Intern Med 1939; 64 : 69-83.

\section{ADRESSES}

C. Mcrlct-Bénichou : directeur de recherche a l'Inserm. T. Gilbert : chargé de recherche à l'Inserm. M. Lclièvre-Pégoricr: chargée de recherche à l'Inserm. Unité de recherche sur le dévcloppement normal et pathologique des fonctions épithćliales. Inserm U. 319, Tour 33-43,

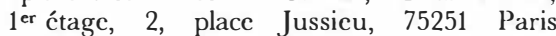
Cedex 5, France.

B. Lcroy : praticien hospitalier, service de nćphrologic pédiatrique, hôpital ArmandTrousscau, 26, avenuc du Doctcur-ArnoldNetter, 75012 Paris, France.

\section{TIRÉS A PART}

\section{Mcrlet-Bénichou.}

